\title{
Passive Avoidance Training and Recall are Associated with Increased Glutamate Levels in the Intermediate Medial Hyperstriatum Ventrale of the Day-Old Chick
}

\author{
Jonathan N. Daisley, ${ }^{1 \dagger}$ Michael Gruss, ${ }^{2}$ Steven P.R. Rose ${ }^{1}$ and Katharina Braun ${ }^{2}$ \\ ${ }^{1}$ Brain Research Group, The Open University, Milton Keynes MK7 6AA UK; \\ ${ }^{2}$ Project Group Juvenile Learning, Leibniz-Institute for Neurobiology, \\ Brenneckestrasse 6, 39118 Magdeburg, Germany
}

\section{SUMMARY}

In the young chick, the intermediate medial hyperstriatum ventrale is involved in learning paradigms, including imprinting and passive avoidance learning. Biochemical changes in the intermediate medial hyperstriatum ventrale following learning include an up-regulation of amino-acid transmitter levels and receptor activity. To follow the changes of extracellular amino acid levels during passive avoidance training, we used an in vivo microdialysis technique. Probes were implanted in chicks before training the animals, either on a methylanthranylate- or water-coated bead. One hour later, recall was tested in both groups by presenting a similar bead. An increase of extracellular glutamate levels accompanied training and testing in both groups; during training, glutamate release was higher in methylanthranylate-trained than in water-trained chicks. When compared with the methylanthranylatetrained chicks during testing, the water-trained chicks showed enhanced extra-cellular glutamate levels. No other amino acid examined showed significant changes. After testing, the chicks were anesthetized and release- stimulated

\footnotetext{
${ }^{\top}$ Corresponding author:

Dr. J.N. Daisley

Lab C-05, Depto de Plasticidad Neural

Instituto Cajal, Avda. Dr. Arce 37

28002 Madrid, SPAIN

Tel: +34-1 585 4741; Fax: +34-1 5854754

e-mail: jonathan@cajal.csic.es
}

with an infusion of $50 \mathrm{mM}$ potassium. Extracellular glutamate and taurine levels were significantly increased in both methylanthranylate- and water-trained chicks. The presentation of methylanthranylate as an olfactory stimulus significantly enhanced glutamate levels, especially in methylanthranylate-trained chicks. The results suggest that such changes in extracellular glutamate levels in the intermediate medial hyperstriatum ventrale accompany pecking at either the water- or the methylanthranylate-bead. The taste of the aversant may be responsible for the greater increases found in methylanthranylate-trained birds.

\section{KEYWORDS}

avian brain, taurine, olfactory learning, neurotransmitter release,

\section{INTRODUCTION}

Chicks peck spontaneously at small bright objects, such as beads. If the bead is made unpleasant by coating it with a bitter tasting substance, the chick will peck once and thereafter avoid similar-looking though neutral-tasting beads. This phenomenon is the basis of the one-trial passive avoidance task in chicks, which was originally introduced by Cherkin (1969). A merit of this paradigm is that memory formation is initiated after a single event, thus allowing precise timing of 
memory-associated events. In addition, the events associated with the training experience itself can be readily dissociated from the processes occurring during memory formation, when the stimulus is no longer present (Rose, 1991). The formation of memory for the passive avoidance task involves a well-documented cascade of biochemical, electrophysiological, morphological events including (a) an increased glucose utilization (Rose \& Csillag, 1985); (b) an increased calcium flux; (c) PKC translocation (Burchuladze et al., 1990); and (d) changes in dendritic spine density (Patel et al., 1988). These events appear to be localized to particular regions of the chick brain. In particular, the left intermediate medial hyperstriatum ventrale (IMHV) appears to be critically involved in learning, as lesions in this area prevent the acquisition of the task (Patterson et al., 1990). The IMHV is regarded as homologous to the mammalian striate cortex and receives inputs from visual auditory somatosensory systems (Bradley et al., 1985; Rose, 1991). Among the early events in the biochemical cascade that are associated with learning and memory formation are changes in neurotransmitter release and binding. Excitatory amino acids are important in a variety of learning models, both in mammals [for example, spatial learning (Morris et al., 1990).] and in birds [imprinting (McCabe \& Horn 1988; Bock et al., 1996)] and have been implicated in passive avoidance learning. Thirty minutes after training on the latter task, the binding of the NMDA receptor is increased (Stewart et al., 1992), whereas injections of MK-801 (a non-competitive NMDA receptor antagonist), either before or up to $5 \mathrm{~min}$ after training, cause amnesia (Burchuladze \& Rose, 1992). A role for the inhibitory transmitter GABA has also been suggested; training results in an enhanced number of GABA receptors (Martijena \& Arce 1994), whereas bilateral injections of the GABA agonist, muscimol, produce amnesia (Clements \& Bourne 1996). We have also shown changes in amino-acid-transmitter (glutamate and GABA) release from slices of IMHV incubated in high potassium media (Daisley \& Rose 1994). The results indicate a critical involvement of glutamate and GABA neurotransmission in the learning and formation of memory for a one-trial passive avoidance task.
Microdialysis, coupled with high performance liquid chromatography (HPLC), is a powerful method for analyzing both local and time-dependent accumulation of neurotransmitters and their metabolites in the extracellular space of awake, freelymoving, naturally behaving animals (Benveniste \& Hüttemeier, 1990). In previous research, (Gruss \& Braun 1996, 1997), changes of transmitters and transmitter metabolites were demonstrated in the chick by microdialysis techniques, using a model of imprinting, wherein chicks were trained to approach an imprinting tone

In this paper we report the use of this microdialysis technique to follow the changes in extracellular concentrations of amino acids, specifically glutamate, following training on the one-trial passive avoidance task.

\section{EXPERIMENTAL}

\section{Animals}

White-leghorn-chicken eggs (Horstmann Nienburg, Germany) were incubated at $37^{\circ} \mathrm{C}$. After hatching, chicks of both sexes were reared in a communal brooder with a day/night cycle of $12 / 12$ $\mathrm{hr}$ and provided freely with food and water The care and use of these animals were performed according to the policy on the use of animals in neuroscience in Germany All protocols were approved by a review committee of the State of Saxony-Anhalt, Germany.

\section{Passive avoidance training procedure}

Training was in accordance with Lössner \& Rose (1983), with one specific alteration: the chicks were not pre-trained. Thus, the training consisted of a single presentation of a chrome bead (4-mm diameter; dipped in either water $(W ; n=6)$ or the bitter-tasting aversant, methylanthranilate (MeA; $\mathrm{n}=6$ ). The chicks that pecked at the MeA-bead exhibited a strong disgust response (shaking of head, wiping of bill, on the floor distress, cheeping. Recall was tested $1 \mathrm{hr}$ after training. The test consisted of a 10-sec presentation of a dry chrome bead identical to that used in training. W-trained birds pecked the bead; MeA-trained birds avoided it. 
In vivo microdialysis

At 24-hr post-hatching, the experimental chick was removed from the brooder and anesthetized by placing it in a container saturated with halothane (4\%). About $15 \mathrm{~min}$ later, the chick was removed from the container, and then the anesthetized animal was mounted in a stereotactic frame; during the surgery halothane $(2 \%)$ was pumped over the nostrils to maintain the chick under anesthesia. A microdialysis probe (CMA 11 ; 1mm; Cuprophan; CMA Micro-dialysis, Sweden) was implanted into the left IMHV and fixed on the skull with dental cement (Harvard Dental Germany). Implantation coordinates were with respect to the y-point AP $+38 \mathrm{~mm} \mathrm{~L}+23 \mathrm{~mm} \mathrm{~V}+21 \mathrm{~mm}$. After the implantation, the animals were returned to a home pen, where they were allowed to recover from the operation for 1-2 hr. The microdialysis probe was perfused with a modified Ringer's solution [120 mM, Na $6 \mathrm{mM}, \mathrm{K}^{+} 2 \mathrm{M}, \mathrm{Ca}^{++} 125 \mathrm{mM}, \mathrm{Mg}^{++}$ $129 \mathrm{M}, \mathrm{Cl}^{-} 125 \mathrm{M}, \mathrm{H}_{2} \mathrm{PO}_{4}^{-} 21 \mathrm{mM}, \mathrm{HCO}_{3}^{-}(\mathrm{pH}$ 7.4)], at a flow rate of $11 \mu \mathrm{l} / \mathrm{min}$, using a microinjection pump (CMA/100, Carnegie Medicin Sweden). After a stabilization period (usually about 2-3 hr), 10-min samples of $11 \mu \mathrm{l}$ each were collected (a) before training for 40 minutes, then (b) during, and (c) subsequent to, training and testing These same four pre-training samples were used as a baseline for both training and testing. Two hr after testing, the chicks were anesthetized [urethane ( $40 \% \mathrm{wt} / \mathrm{vol}$ ) $0.65 \mathrm{ml} / 100 \mathrm{~g}$ body weight], with concurrent sampling of the microdialysates. Urethane administration resulted in a long-lasting anesthesia. After the baseline had reached a stable value following the administration of the anaesthetic (approximately 1-1.5 hr later), the first Ringer's solution was switched for $30 \mathrm{~min}$ to a second modified Ringer's solution containing $50 \mathrm{mM}$ potassium (to maintain osmolarity, the sodium ion concentration was lowered accordingly). After this treatment, the perfusion medium was switched back to the first Ringer's solution, and more samples were collected. After a return to a steady baseline toward the end of the experimental period (from 6.5-7 hr), the anesthetized chicks were first presented with a dry tissue, which was placed close to the anesthetized chick's nostrils for a 10-min sampling period The tissue was then removed, and $40 \mathrm{~min}$ were allowed to elapse before the tissue (this time soaked in MeA) was again placed for 10 min in a similar position, close to the nostrils of the chick. Both groups of chicks (W- and MeA-trained) received $\mathrm{MeA}$ as the olfactory stimulus.

As is standard practice in these laboratories, each day the microdialysis experiments were performed at essentially the same hour (starting between 10:30 and 11:30 am) to reduce the effect of diurnal rhythms on the levels of transmitters present in the microdialysates.

\section{Histology}

To verify the position of the probe serial sections, $(40 \mu \mathrm{m}$; were taken from the brain of each animal in an area near the insertion point of the probe. The sections were counterstained with cresyl violet (Nissl stain), and then the location of the probe was determined. If the probe was not located within the IMHV, the data from such chicks were discarded.

\section{Amino acid analysis}

The microdialysates were analyzed for aminoacid content, using a method adapted from Lindroth \& Mopper (1979) and later developed by Gruss \& Braun (1996). In brief, the modified method involved an HPLC system equipped with a C-18 reversed-phase column (LiChrospher RP18; Merck, Germany). The column was eluted using a nonlinear gradient from $13 \%$ to $100 \%$ methanol in $01 \mathrm{M}$ phosphate buffer ( $\mathrm{pH}$ 7.4). After automatic precolumn derivatization with o-phthaldialdehyde (oPA; Sigma, Germany), the eluent was monitored using fluorescence detection Chromatograms were analyzed using a PC-based, HPLC Manager program (Merck Germany).

\section{Data analysis}

As described in a previous work (Gruss \& Braun, 1996) considerable variation between individual chicks was found in the levels of amino acids recorded, with basal-level measurements for glutamate varying up to ten-fold For this reason, all measurements were compared with the average of four baseline/pre-treatment values (the average was assigned a value of $100 \%$ ). Therefore, all traininginduced changes in amino-acid levels were 
calculated as mean \pm SEM percentages of the basal release The basal release was determined as the average of the four samples (set at $100 \%$ ) that were taken immediately before training. Statistical analysis of the data was carried out using Friedman's-ANOVA, followed by the Wilcoxonmatched pairs test for within-group measurements or by the Mann-Whitney U-test for comparisons between groups. All statistics were considered significant when $\mathrm{p}=<0.05$.

\section{RESULTS}

\section{Basal level measurements}

For pre-training microdialysates, basal concentrations, indicated as mean \pm SEM values (in $\mu \mathrm{M}$ ) for glutamate, aspartate, taurine, glutamine, and glycine, are presented in Table 1.

\section{TABLE 1}

Basal concentrations of amino acids in chick IMHV dialysates ${ }^{1}$

\begin{tabular}{ll}
\hline Glutamate & $0.84 \pm 0.15$ \\
Aspartate & $0.05 \pm 0.01$ \\
Taurine & $8.5 \pm 1.23$ \\
Glutamine & $2.03 \pm 0.33$ \\
Glycine & $0.41 \pm 0.08$ \\
\hline
\end{tabular}

${ }^{1}$ The data represent the mean \pm SEM values (in $\mu \mathrm{M}$ ) and are not corrected for in-vitro recoveries of microdialysis probes.

In general, the levels of amino acids were similar to those found previously in the chick forebrain (Gruss \& Braun 1996). Basal levels of extracellular GABA were not detectable in all experiments, which agrees with previous data from other microdialysis studies (Qume et al., 1995; Gruss \& Braun, 1996). This result may be due in part to the oPA-derivatization technique, which yields highly unstable derivatives of GABA, with a half-life time of only $4 \mathrm{~min}$ (Lasley, et al 1984).

2. Training-/testing-induced changes in glutamate release

Chicks were trained and tested as described in the Experimental section. Six chicks were used in each group. For both training and testing values, the pre-training values were taken as a baseline for comparison.

Training chicks to peck a bead coated either in $\mathrm{W}$ - or in MeA elicited an increase in extracellular glutamate levels from the left IMHV $(p<0.001$ Friedman's ANOVA; Fig 1. and Table 2). When compared with W-trained chicks, the MeA-trained chicks had significantly elevated levels of glutamate ( $p<0.05$, Mann-Whitney U-test).

By 10 min after training, the level of glutamate fell in both groups, although the post-training levels continued to be higher than the baseline values ( $p<0.05$, Friedman's ANOVA). When the chicks were tested, both groups again showed increased glutamate levels $(p<0.05$, Friedman's-ANOVA). The W-trained chicks now showed higher levels of glutamate than did MeA-trained chicks $(p<005$, Mann-Whitney U-test).

In both groups, aspartate, taurine, glutamine, and glycine showed no change during training and testing (Table 2).

\section{Effect of potassium stimulation on in vivo glutamate accumulation}

Two hr after testing, the chicks were anesthetized with urethane. During the handling and injection of the anesthetic, a significant increase in glutamate release, which persisted for up to $30 \mathrm{~min}$, was found (results were consistent in the next three microdialysates taken after handling; $p<0.05$, Friedman's-ANOVA) (data not shown). No difference was observed between the groups ( $p>0.10$, Mann-Whitney U-test). After the glutamate levels had stabilized (a further 1.5-2 hr), a new baseline was taken. When the new baseline points were compared with the original four that were taken before training, no difference was found between the new baselines of the two groups ( $p>0.05$, Friedman's-ANOVA). The perfusate was then changed to the second Ringer's solution containing $50 \mathrm{mM}$ potassium, which was maintained 
TABLE 2

Changes in extracellular amino acids following experimental manipulations ${ }^{1}$

\begin{tabular}{|c|c|c|c|c|c|c|}
\hline Amino acid & Bead coating & Training & Testing & Potassium & No odor & Odor \\
\hline \multirow[t]{2}{*}{ Glutamate } & W & $184 \pm 31$ & $286 \pm 36$ & $331 \pm 36$ & $121 \pm 34$ & $202 \pm 23$ \\
\hline & $\mathrm{MeA}$ & $253 \pm 33^{*}$ & $223 \pm 33 *$ & $422 \pm 48 *$ & $126 \pm 38$ & $272 \pm 33^{*}$ \\
\hline \multirow[t]{2}{*}{ Aspartate } & W & $114 \pm 33$ & $121 \pm 48$ & $185 \pm 89$ & $98 \pm 35$ & $123 \pm 39$ \\
\hline & MeA & $108 \pm 37$ & $102 \pm 49$ & $167 \pm 70$ & $113 \pm 37$ & $131 \pm 44$ \\
\hline \multirow[t]{2}{*}{ Taurine } & W & $110 \pm 21$ & $123 \pm 27$ & $324 \pm 57$ & $109 \pm 14$ & $109 \pm 14$ \\
\hline & $\mathrm{MeA}$ & $113 \pm 23$ & 11819 & $383 \pm 65$ & $98 \pm 16$ & $119 \pm 24$ \\
\hline \multirow[t]{2}{*}{ Glutamine } & W & $124 \pm 25$ & $121 \pm 21$ & $123 \pm 27$ & $112 \pm 19$ & $116 \pm 17$ \\
\hline & $\mathrm{MeA}$ & $123 \pm 24$ & $120 \pm 25$ & $121 \pm 24$ & $106 \pm 13$ & $121 \pm 22$ \\
\hline \multirow[t]{2}{*}{ Glycine } & $\mathrm{W}$ & $122 \pm 32$ & $120 \pm 44$ & $121 \pm 38$ & $94 \pm 38$ & $109 \pm 29$ \\
\hline & $\mathrm{MeA}$ & $131 \pm 33$ & $128 \pm 50$ & $130 \pm 47$ & $101 \pm 47$ & $118 \pm 42$ \\
\hline
\end{tabular}

${ }^{1}$ Data are mean \pm standard deviation values, expressed as a percentage of baseline values $100 \%$; Figures in boldface indicate values significantly higher than baseline $(\mathrm{p}<.005$ Wilcoxon test; whereas a * indicates a difference between W- and MeA-trained chicks $(\mathrm{p}<.005$ Mann-Whitney Utest;

\section{Effect of training and testing on extracellular glutamate in IMHV}

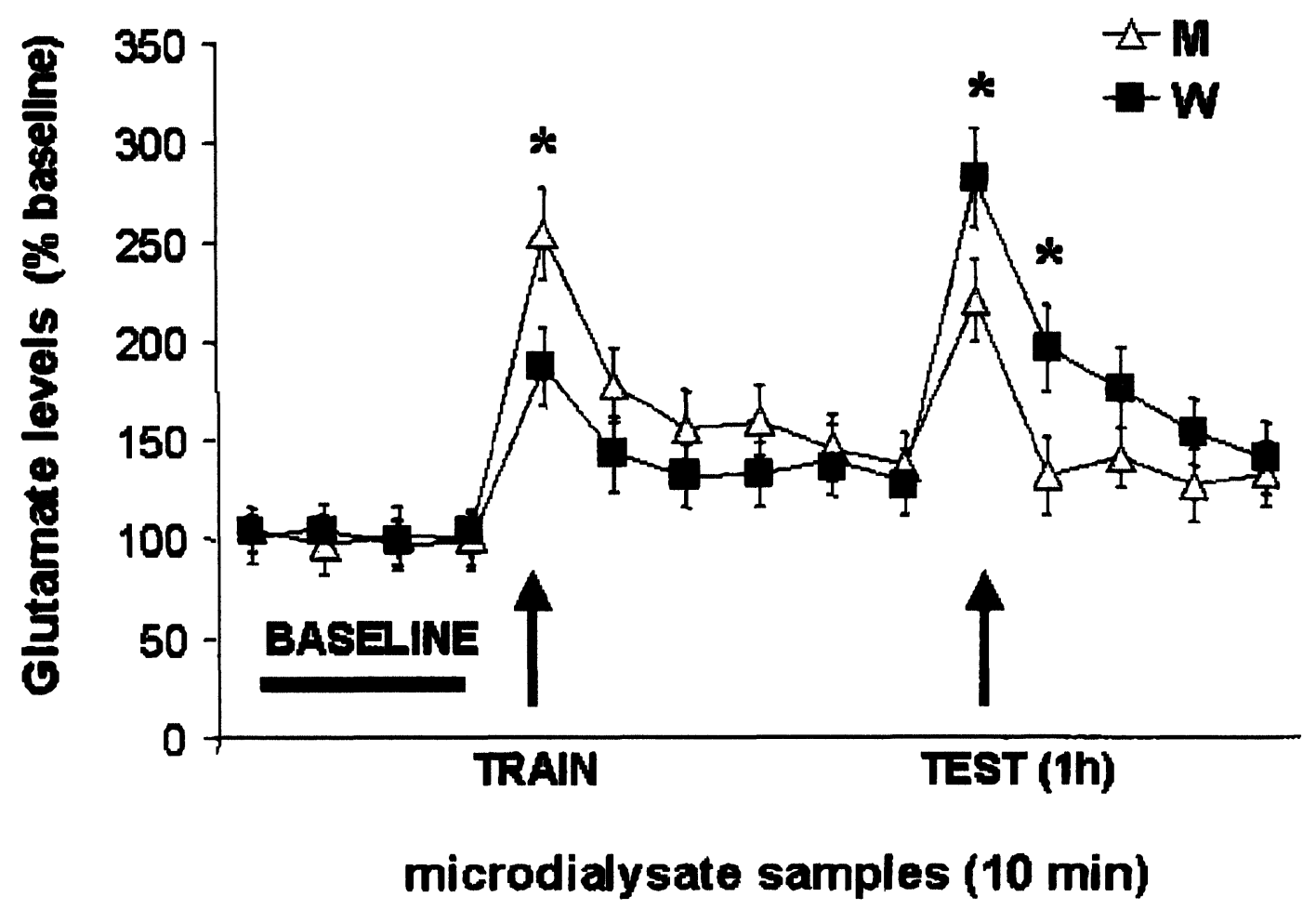

Fig 1: Effect of passive avoidance training and subsequent testing on the extracellular accumulation of glutamate in the left IMHV of MeA- (M) and W-trained (W) chicks. Data are mean \pm SEM (bars) values. The arrows indicate the dialysate samples, including times of bead presentation on training and testing. ${ }^{*} \mathrm{p}<.005$ between groups (Mann-Whitney U-test). 


\section{Effect of potassium stimulation on glutamate levels in the IMHV}

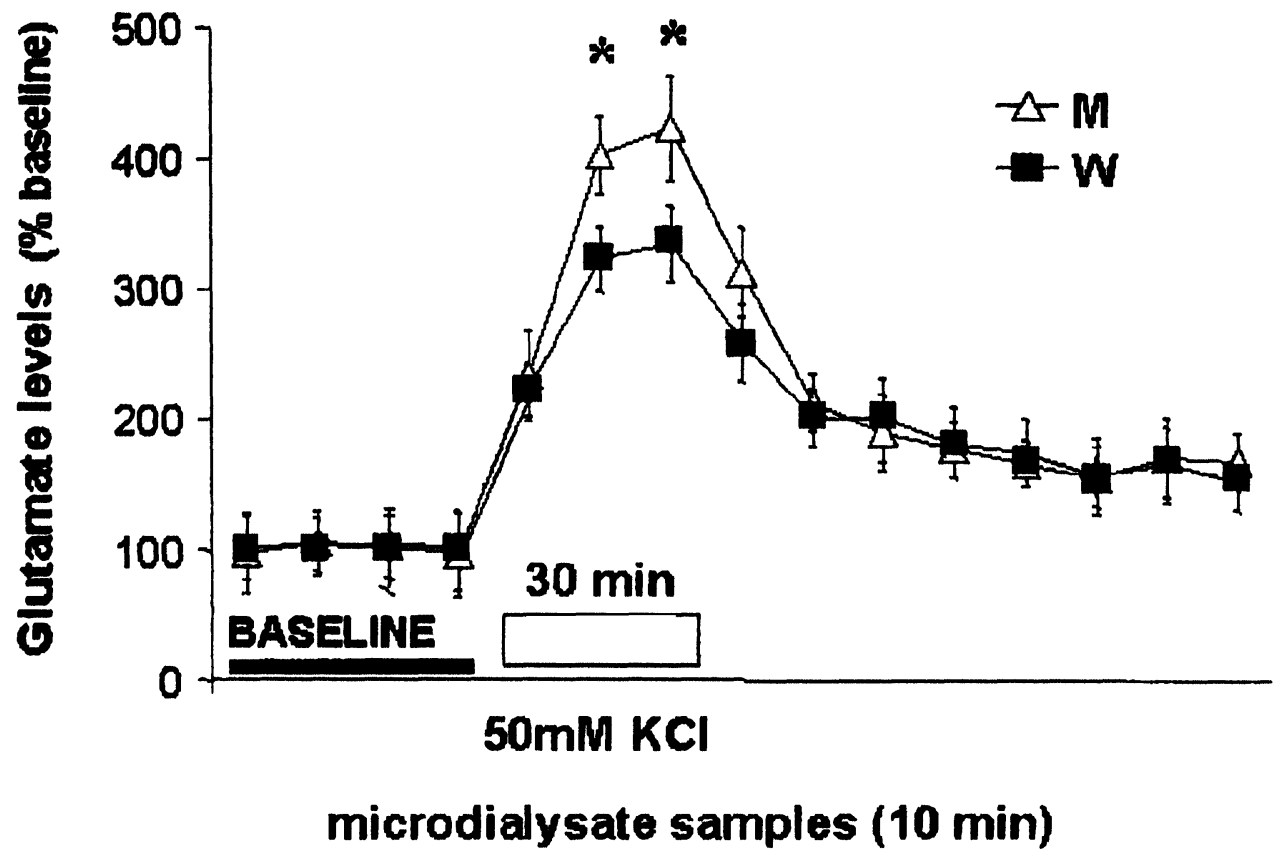

Fig 2: The effect of potassium stimulation on in vivo glutamate release from the left IMHV of MeA- (M) and W-trained (W) chicks. Data are mean \pm SEM (bars) values. The horizontal bar indicates the 30 min of stimulation with $50-\mathrm{mM}$ potassium. ${ }^{*} \mathrm{p}<0.05$ between groups (Mann-Whitney U-test).

\section{Effect of M-odour presentation on glutamate levels in IMHV}

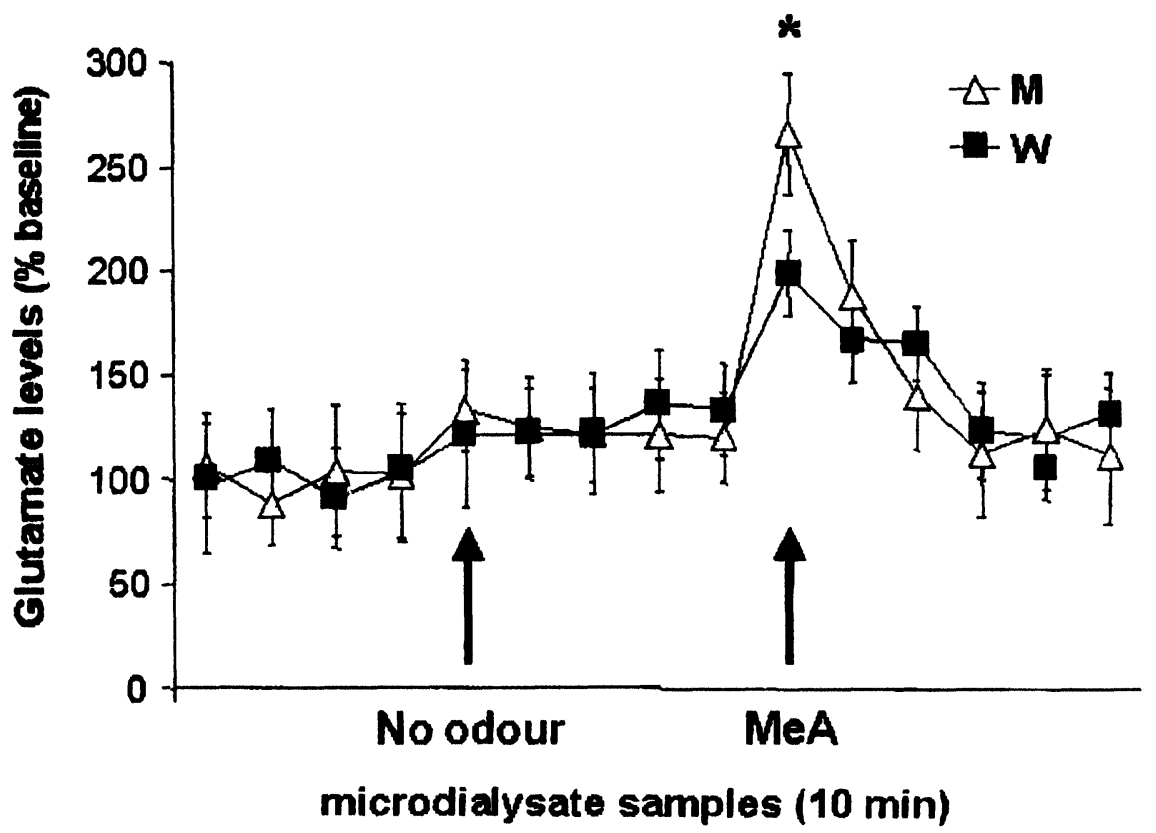

Fig 3: The effect of MeA presentation as an odor to anaesthetized MeA- (M) and W-trained (W) chicks on in vivo glutamate levels in the left IMHV. Data are mean \pm SEM (bars) values. The arrows indicate the microdialysate samples, including the times of dry tissue or MeA-soaked tissue presentation. ${ }^{*} \mathrm{p}<0.05$ between groups (Mann-Whitney U-test). 
for three samples (30 $\mathrm{min})$, and then returned to the first Ringer's solution. In both W- and MeA-trained chicks, switching to potassium-ion-stimulation increased the glutamate levels to above baseline values $(p<0.05$, Friedman's-ANOVA; see Fig. 2 and Table 2). When compared with that of the W-trained birds, the glutamate release was significantly higher in the IMHV of the MeA-trained group ( $p<0.05$ Mann-Whitney U-test). Increased glutamate levels were also found in the four subsequent microdialysis samples (up to $40 \mathrm{~min}$ post-testing) in both groups ( $p<0.05$, Friedman'sANOVA). In addition, taurine was elevated above baseline levels ( $p<0.05$, Friedman's-ANOVA), but no differences were apparent between the two experimental groups ( $p>.005$ Mann-Whitney Utest).

Under these conditions, extracellular GABA levels were measurable, indicating an increase in extracellular GABA during stimulation with $50 \mathrm{mM}$ potassium, but no differences were found between the W- and MeA-trained chicks ( $p>0.10$, MannWhitney U-test). Because this increase cannot be referred back to a baseline value as with the other amino acids, the potassium-induced values are not indicated in Table 2 . No changes in the extracellular levels of aspartate, glutamine, or glycine were observed in either experimental group during infusion of $50 \mathrm{mM}$ potassium (Table 2).

\section{The effect of olfactory stimulation with MeA on glutamate release}

Around $2 \mathrm{hr}$ after the end of potassium stimulation, the chicks (still under anesthetic) were presented sequentially with (a) a dry tissue for 10 min and (b) $40 \mathrm{~min}$ later, a tissue soaked for $10 \mathrm{~min}$ in MeA (for $10 \mathrm{~min}$ ) tissue (Table 2 and Fig. 3). Glutamate levels in the microdialysates from both groups showed no change from the new baseline (four samples taken before the presentation of the tissue) when presented with the dry, odorless tissue ( $p>0.10$, Friedman's-ANOVA). The MeA-soaked tissue, however, elicited an increase in glutamate levels in both groups $(p<0.05$, Friedman'sANOVA). This increase was significantly higher in the chicks that had been previously trained on the MeA-bead ( $p<0.05$, Mann-Whitney U-test).

In both groups, no change in the extracellular levels of aspartate, taurine, glutamine, or glycine were measured during the presentation of either the dry or the MeA-soaked tissue (Table 2).

\section{DISCUSSION}

Using microdialysis in the freely behaving chick, we could follow the changes in extracellular levels of glutamate and other amino acids that are associated with the presentation of a bead coated in the bitter-tasting substance methylanthranilate (MeA). Pecking at a bead, whether coated in water (W) or in the aversant, resulted in increases in glutamate accumulation; offering the bead to trained birds at test, whether pecking or not, also resulted in an increase. The MeA-chicks, however, showed an enhanced glutamate accumulation when compared with W-chicks on training, whereas on testing, W-chicks (which pecked) had a significantly higher level of glutamate than did MeA-chicks (which avoided the bead). Stimulation of urethaneanesthetized birds with $50 \mathrm{mM}$ potassium perfusion also produced enhanced glutamate accumulation; such stimulation was higher in the MeA-birds That the odor of the MeA is of itself sufficient to produce a reminder response was indicated by the finding that in the MeA-trained chicks, the presentation of the aversant next to the anesthetized chick's nostrils produced an increase in glutamate levels.

Both groups of chicks showed an enhanced glutamate release that was associated with pecking a bead during training. Because both $\mathrm{MeA}-$ and W-training results in learning (in the former case aversive learning and in the latter case neutral or appetitive learning), that pecking should elicit a response in the W-birds is perhaps not surprising. Nevertheless, the larger effect in the MeA-trained birds presumably relates to a combination of factors; both to the taste itself and to the greater salience of the aversive experience. That pecking itself (which involves visual acuity motor coordination and/or arousal) is sufficient to produce an increase in glutamate levels is indicated by the finding that on testing, the W-birds that did peck showed a greater increase than did the MeA-birds.

Potassium stimulation for a sustained period (30 min) produced an expected increase in glutamate release. In addition, this result also provides evidence that the IMHV is an area involved in longer-term processes because the induced release 
from the MeA-trained chicks was significantly higher than that in the W-trained birds. We believe that this increase is related to memory events. In a previous study (Daisley \& Rose 1994), using slices of IMHV, we could not show enhanced glutamate release to potassium stimulation in the left IMHV in vitro, neither at $3 \mathrm{hr}$ nor at $65 \mathrm{hr}$ after training $(2 \mathrm{hr}$ or $5.5 \mathrm{hr}$ after testing). This discrepancy may be due to the different methods used: Removing tissue on ice after training and then pre-incubating the material for a considerable period ( $1 \mathrm{hr}$ ) is likely to produce results that are different from the in vivo assay carried out in the present study. Using an in vivo preparation enables the continued input from other brain regions into the IMHV, therefore the restructuring of synaptic and dendritic material, which obviously are essential processes in learning and ongoing memory formation, can proceed.

Glutamate, however, is not the only amino acid whose accumulation changes as a result of potassium stimulation. A reliable increase of extracellular taurine levels was observed, with no difference between the experimental groups. Such a potassium-induced elevation is well documented in the mammalian literature (Westerink et al., 1987; Fujikawa et al., 1996) and was also observed by us in previous experiments using chicks (Gruss \& Braun, 1996). The reasons for the increased taurine levels may be multi-fold and may be related to its assumed role as an osmoregulator (Solis, et al., 1988; Wade, et al., 1988).

The presentation of methylanthranilate to the anesthetized chicks as an olfactory stimulus resulted in increased glutamate accumulation in both MeAand W-birds; nevertheless, the significantly larger response in the MeA-trained birds indicated that memory for the aversive taste must play an important part. Thus, re-presenting the stimulus, although only alerting olfactory-associated activity, appears to be sufficient to produce IMHV activity. The olfactory bulb of birds is thought to have connections, via a medial route, to areas of the hyperstriatum (Rieke \& Wenzel, 1978). This would provide a route for olfactory-based learning. Recently, associating a bead-peck merely with the odor of MeA has been demon-strated to be sufficient to produce an avoidance of the test bead 10 min after training (Burne \& Rogers, 1997), again suggesting that our results are related to the learning element in the task. Because of their training regime, however, a greater likelihood of glutamate elevation in the MeA-trained chicks cannot be ruled out because we did not control for this factor by using a neutral olfactory stimulus. The small effect in the W-trained birds does suggest that an olfactory stimulus may up-regulate the general activity and efficacy of olfactory pathways.

In conclusion, we suggest that the changes in extracellular glutamate levels that we found on training and testing are associated in part with nonspecific concomitants of the training and testing experience rather than with memory formation per se. That increased glutamate was also found during handling and injection of the anesthetic (a stressful event) would also imply that the IMHV is involved in or is affected by such arousal The greater changes found in the MeA-trained birds, however, and the differences in their responses, both on testing and on presentation of the MeA-odor, suggest that at least part of the enhanced glutamate accumulation is indeed related to memory formation. The studies presented here suggest, therefore, that use of the microdialysis technique can provide valuable information about the spatio-temporal dynamics of neurotransmitter involvement in the synaptic plasticity that is associated with memory formation.

\section{ACKNOWLEDGEMENT}

These experiments were conducted between our laboratories under the ESF-ENP sponsored collaborative programme on neural mechanisms of learning and memory

\section{REFERENCES}

Benveniste H, Hüttemeier PC. Microdialysis-theory and application. Prog Neurobiol 1990; 35: 195-215.

Bock J, Wolf A, Braun K. Influence of the N-methyl-Daspartate receptor antagonist DL-2-amino-5-phosphonovaleric acid on auditory filial imprinting in the domestic chick. Neurobiol Learn Mem 1996; 65: 177 188.

Bradley P, Davies DC, Horn G. Connections of the hyperstriatum ventrale of the domestic chick (Gallus domesticus). J Anat 1985; 140: 577-589.

Burchuladze R, Rose SPR. Memory formation in day-old chicks requires NMDA but not non-NMDA glutamate receptors. Eur J Neurosci 1992; 4: 533-538. 
Burchuladze R, Potter J, Rose SPR. Memory formation in the chick depends on membrane-bound protein kinase C. Brain Res 1990; 535: 131-138.

Burne TH, Rogers LJ. Relative importance of odour and taste in the one-trial passive avoidance learning bead task. Physiol Behav 1997; 62: 1299-1302.

Cherkin A. Kinetics of memory consolidation. Role of amnestic treatment parameters. Proc Natl Acad Sci USA 1969; 63: 1094-1101.

Clements MP, Bourne RC. Passive avoidance learning in the day-old chick is modulated by GABAergic agents. Pharmacol Biochem Behav 1996; 53: 629-634.

Fujikawa DG, Kim JS, Daniels AH, Alcaraz AF, Sohn TB. In vivo elevation of extracellular potassium in the rat amygdala increases extracellular glutamate and aspartate and damages neurons. Neuroscience 1996; 3: 695-706.

Daisley JN, Rose SPR. The effect of a passive avoidance task on the release of amino acids in vitro from the left intermediate medial hyperstriatum ventrale (IMHV) of the day-old chick. Biochem Soc Trans 1994; 22: 160S.

Gruss M, Braun K. Stimulus-evoked increase of glutamate in the mediorostral neostriatum/hyperstriatum ventrale of domestic chick after auditory imprinting: an in vivo microdialysis study. J Neurochem 1996; 66: 11671173.

Gruss M, Braun K. Distinct activation of monoaminergic pathways in chick brain in relation to auditory imprinting and stressful situations: a microdialysis study. Neuroscience 1997; 76: 891-899.

Lasley SM, Greenland RD, Michaelson IA. Determination of $\gamma$-aminobutyric and glutamic acids in rat brain by liquid chromatography with electrochemical detection. Life Sci 1984; 35: 1921-1930.

Lindroth P, Mopper K. High performance liquid chromatoraphic determination of sub-picomole amounts of amino acids by precolumn fluorescence derivatization with o-phthaldialdehyde. Anal Chem 1979; 51: 16671674.

Lössner B, Rose SPR. Passive avoidance training increases fucokinase activity in the right forebrain tissue of day-old chicks. J Neurochem 1983; 41: 1357-1363.

Martijena ID, Arce A. Transient benzodiazepene-GABA receptor increase after a passive avoidance learning in synaptosomal membranes from chick forebrain. Can J Physiol Pharmacol 1994; 72: 233-237.
McCabe BJ, Horn G. Learning and memory: regional changes in $\mathrm{N}$-methyl-D-aspartate receptors in chick brain after imprinting. Proc Natl Acad Sci USA 1988; 85: 2849-2853.

Morris RGM, Davis S, Butcher SP. Hippocampal synaptic plasticity and NMDA receptors: a role in information storage. Phil Trans Royal Soc 1990; 329: 187-204.

Patel SN, Rose SPR, Stewart MG. Training-induced spine density changes are specifically related to memory formation processes in the chick, Gallus domesticus. Brain Res 1988; 449: 34-46.

Patterson TA, Gilbert DB, Rose SPR. Pre- and posttraining lesions of the intermediate medial hyperstriatum ventrale and passive avoidance learning in the chick. Exp Brain Res 1990; 80: 189-195.

Qume M, Whitton PS, Fowler LJ. The effect of chronic treatment with the GABA transaminase inhibitors $\gamma$-vinyl-GABA and ethanolamine-O-sulphate on the in vivo release of GABA from rat hippocampus. $\mathrm{J}$ Neurochem 1995; 64: 2256-2261.

Rieke GK, Wenzel BM. Forebrain connections of the pigeon olfactory bulb. J Morphol 1978; 158: 41-56.

Rose SPR. Biochemical mechanisms involved in memory formation in the chick. In: Andrew RJ, ed, Neural and Behavioural Plasticity: The Use of the Domestic Chick as a Model. Oxford: Oxford University Press, 1991; 277-304.

Rose SPR, Csillag A. Passive avoidance training results in lasting changes in deoxyglucose metabolism in left hemisphere regions of chick brain. Behav Neural Biol 1985; 44: 315-324.

Solis JM, Herranz AS, Herreras O, Lerma J, Martin del Rio R. Does taurine act as an osmoregulatory substance in the rat brain? Neurosci Lett 1988; 91: 53-58.

Stewart MG, Bourne R, Steele RJ. Quantitative autoradiographic demonstration of changes in binding to NMDA-sensitive $\left[{ }^{3} \mathrm{H}\right]$-glutamate and $\left[{ }^{3} \mathrm{H}\right]-\mathrm{MK}-801$, but not [3H]-AMPA receptors, in chick forebrain 30 minutes after passive avoidance training. Eur J Neurosci 1992; 4: 936-943.

Wade JV, Olson JP, Samson FE, Nelson SR, Pazdernik TL. A possible role for taurine in osmoregulation within the brain. J Neurochem 1988; 51: 740-745.

Westerink BHC, Damsma G, Rollema H, de Vries JB, Horn AS. Scope and limitations of in vivo brain dialysis: a comparison of its application to various neurotransmitter systems. Life Sci 1987; 41: 1763-1776. 

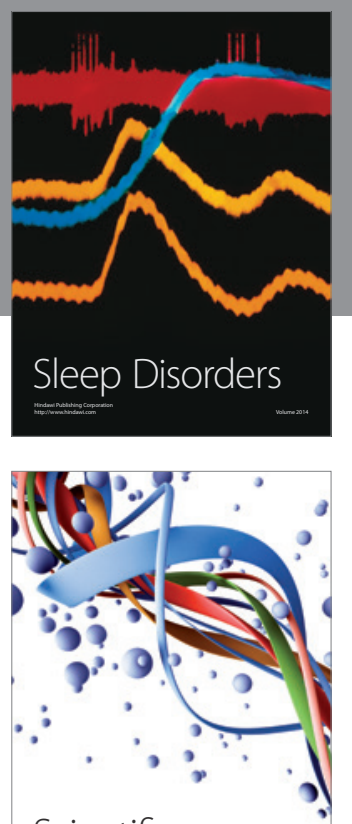

Scientifica
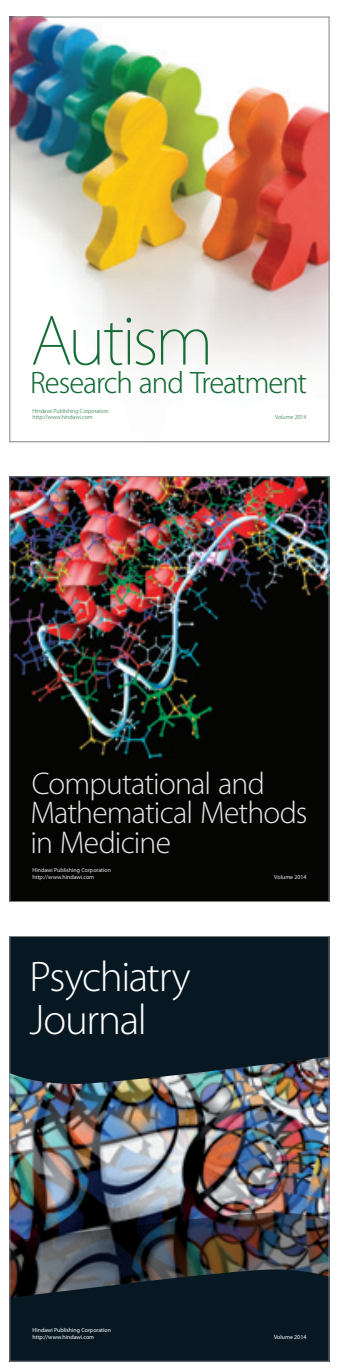
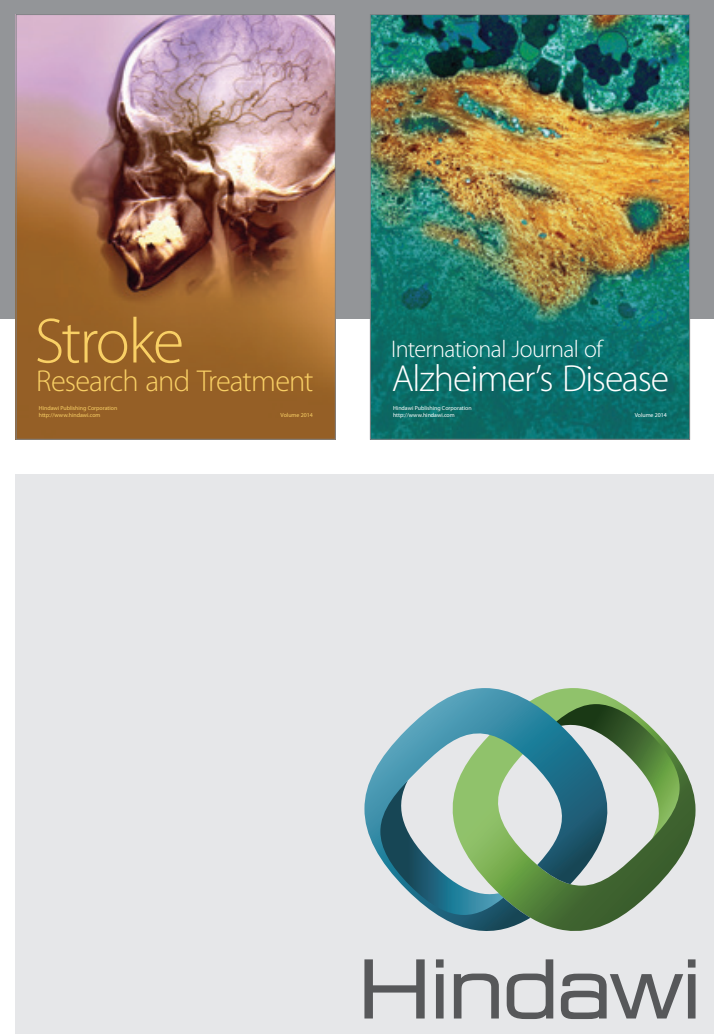

Submit your manuscripts at

http://www.hindawi.com
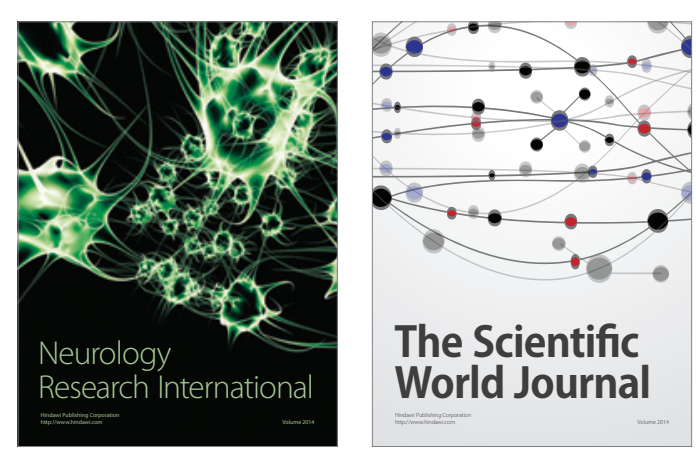

The Scientific World Journal

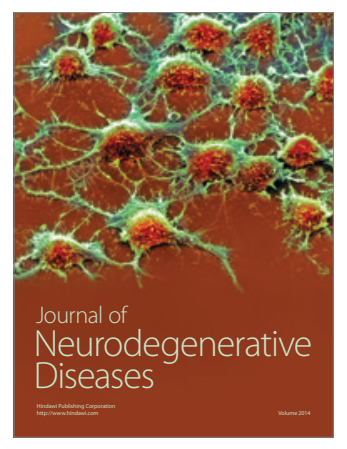

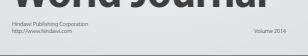

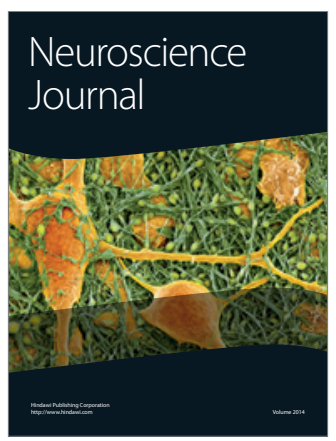

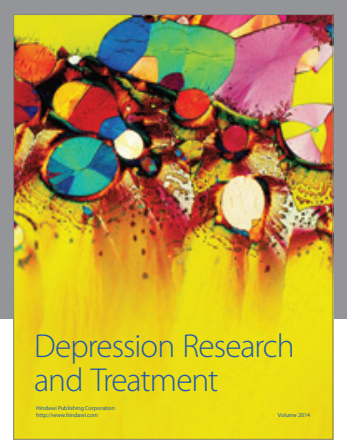
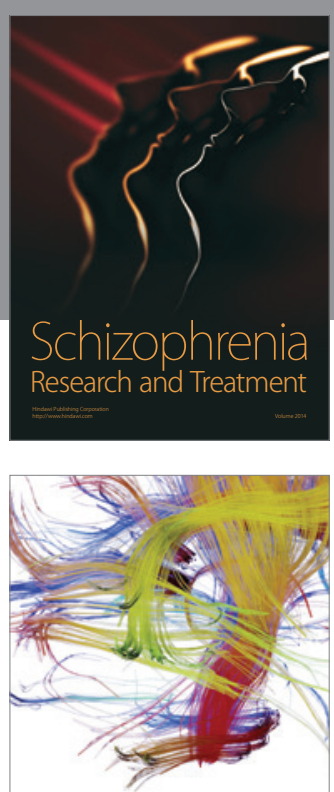

Brain Science

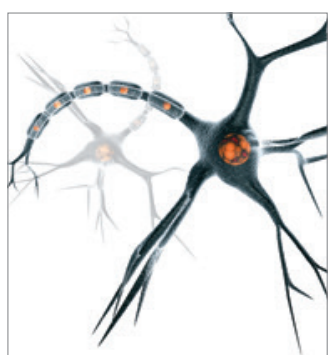

Neural Plasticity
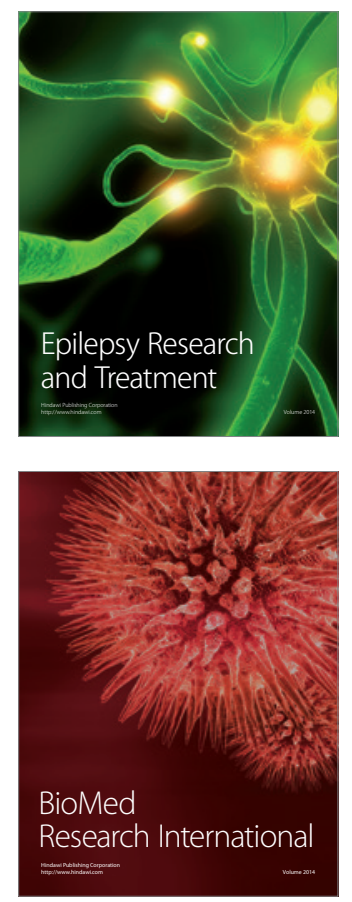

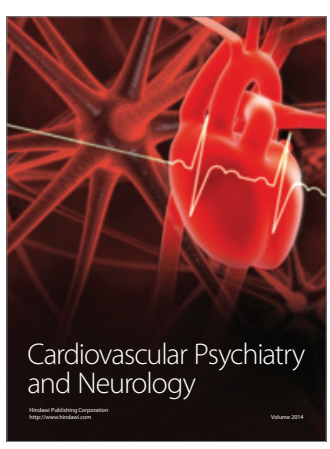

Parkinson's

Disease
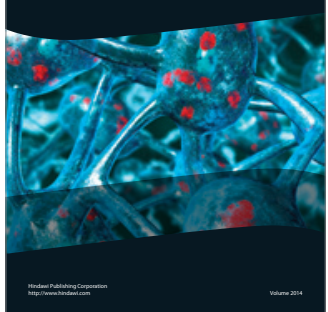